\title{
Ansiedad y estrategias de afrontamiento
}

\author{
Alba Andreo ${ }^{1}$, Pascual Salvador Hilario ${ }^{2}$, y Francisco Javier Orteso ${ }^{3}$ \\ ${ }^{1}$ Máster Psicología General Sanitaria; ${ }^{2}$ Centro de Salud Mental y \\ Drogodependencias de Lorca (España); ${ }^{3}$ Universidad Católica de Murcia (España)
}

\begin{abstract}
La ansiedad es uno de los trastornos psiquiátricos más frecuentes. El afrontamiento hace referencia a las distintas formas con las que las personas se enfrentan a situaciones estresantes. Las investigaciones señalan que, a mayor ansiedad, menor uso de estrategias de afrontamiento (EA) adaptativas y mayor uso de EA desadaptativas. El objetivo general es determinar si existe relación entre la ansiedad y las EA. Se han utilizado dos muestras de participantes, con un muestreo incidental: población clínica (PC) diagnosticada de un trastorno de ansiedad $(n=35)$ y población no clínica (PNC) sin diagnóstico de ansiedad ( $n=38)$. Los instrumentos administrados fueron: un cuestionario sociodemográfico, la Escala de Ansiedad y Depresión de Goldberg y el Inventario de Estrategias de Afrontamiento. Se encontraron diferencias significativas en el uso de las EA adaptativas, la PC empleaba menos que la PNC este tipo de estrategias. Además, la estrategia de reestructuración cognitiva aportó diferencias significativas, siendo menos empleada por la PC que por la PNC. La PC se dividió en dos: uno con sintomatología depresiva y otro sin ella, siendo significativamente mayor el uso de EA desadaptativas en el primer grupo respecto al segundo. En conclusión, los pacientes que presentan un trastorno de ansiedad emplean menos que la población sin diagnóstico las EA adaptativas, especialmente la reestructuración cognitiva.
\end{abstract}

Palabras clave: Ansiedad, estrategias de afrontamiento, estrategias de afrontamiento adaptativas, reestructuración cognitiva.

Anxiety and coping strategies. Anxiety is one of the most frequent psychiatric disorders. Coping refer to the different ways in which people face stressful situations. Research indicates that the greater the anxiety, the less use of adaptive strategies and the greater use of maladaptive strategies. The general objective is to determine if there is a relationrelate between anxiety and coping strategies. Two samples of participants have been used, with an incidental sample: clinical population (PC) diagnosed with an anxiety disorder $(n=35)$ and non-clinical population (PNC) not attended to psychologically treatment or diagnosed with anxiety $(n=38)$. The instruments administered were a sociodemographic questionnaire, the Goldberg Anxiety and Depression Scale and the Inventory of Coping Strategies. Regarding the results, significant differences were found in the use of adaptive or active strategies, the PC used this type of strategies less than the PNC. In addition, the strategy of cognitive restructuring, contributed significant differences, being less used by the PC than by the PNC. The PC was divided into two: one with depressive symptomatology and the other without it, the use of maladaptive coping strategies in the first group being significantly greater than the second. In conclusion, patients with an anxiety disorder use adaptive strategies less than the population without diagnosis, especially cognitive restructuring.

Keywords: Anxiety, coping strategies, adaptative strategies, cognitive restructuring.

Correspondencia: Alba Andreo Valenzuela. Camino Aleurrosas s/n, Apartado de correos 115. C.P.: 30850. Totana, Murcia (España). E-mail: albaandval@gmail.com 
La ansiedad es una respuesta de anticipación ante una amenaza próxima (Asociación Americana de Psiquiatría, APA, 2013). Se trata de una respuesta adaptativa y que todas las personas sienten en mayor o menor grado (Sierra, Ortega, y Zubeidat, 2003). Para diferenciar la ansiedad normal de la ansiedad patológica, la APA (2013) expone que esta última persiste más en el tiempo y se caracteriza por una sobreestimación exagerada del peligro.

Los trastornos de ansiedad son los trastornos psiquiátricos que se presentan con mayor frecuencia en atención primaria y especializada, junto con los trastornos depresivos (Herrero, 2016). En un estudio realizado en el Centro de Salud Mental de Lorca se obtuvo que, de 3006 pacientes, el 40.9\% presentaban diagnósticos de ansiedad según la CIE-10 (Ponce, Hilario, y Orteso, 2018).

Por otra parte, el manejo o afrontamiento hace referencia a la forma en que las personas resuelven o se enfrentan a situaciones estresantes de su vida (Lazarus, 2000). Las estrategias de afrontamiento se relacionan con dos procesos: la evaluación cognitiva y el afrontamiento. La evaluación cognitiva es un proceso por el que la persona realiza un juicio sobre la situación y sus capacidades de afrontamiento (Clark y Beck, 2012).

Por su parte, el afrontamiento se define como los esfuerzos de tipo cognitivo y conductual para resolver demandas internas o externas que la persona evalúa como excesivas en relación a sus recursos, y que además están en constante cambio (Lazarus y Folkman, 1984).

Existen dos modos de referirse al afrontamiento, los estilos y las estrategias (Cano, Rodríguez, y García, 2007). En relación a las primeras, para las que estos autores citan a Pelechano, se trataría de un modo disposicional y estable de afrontar el estrés, y las segundas serían formas más específicas y contextuales de hacerlo.

Lazarus y Folkman, en Lazarus (2000), realizan una clasificación de las estrategias en función de si son orientadas al problema u orientadas a la emoción. Haciendo referencia a la evaluación de las estrategias de afrontamiento, Lazarus (2000) plantea que el estudio de las mismas debería realizarse de forma detallada y con una descripción de lo que piensa y hace la persona ante la situación estresante, así, a finales de los años 70, Lazarus y sus colaboradores comenzaron a elaborar escalas para evaluar estos aspectos.

Jauregui, Herrero-Fernández, y Estévez (2016) señalan que el Inventario de Estrategias de Afrontamiento (Coping Strategies Inventory, CSI) elaborado por Tobin, Holroyd, Reynolds, y Wigal (1989), es una herramienta de referencia para evaluar el afrontamiento con una estructura jerárquica con factores primarios, secundarios y terciarios. 


\section{Estrategias de afrontamiento y su relación con la ansiedad}

En cuanto a investigaciones realizadas con una muestra de pacientes con trastornos de ansiedad, Pozzi et al. (2015) hallaron que la psicopatología se relacionaba con las estrategias de evitación, infiriendo que éstas podrían ser formas ineficaces de afrontamiento. Shrestha (2015), encontró en su estudio que las estrategias de afrontamiento más utilizadas en pacientes con trastorno de ansiedad generalizada fueron las estrategias de aceptación pasiva y estrategias centradas en el problema.

Existen investigaciones que realizan comparaciones de estrategias de afrontamiento en dos grupos, un grupo presentando ansiedad clínica y el otro no. De esta forma, Gantiva, Dávila, y Salgado (2010), empleando una muestra de 60 sujetos, realizaron una comparación de las medias de puntuaciones y los resultados mostraron que las personas con ansiedad, utilizaban significativamente menos las estrategias de reevaluación positiva y solución de problemas; y por otro lado, utilizaban más las estrategias de reacción agresiva, expresión de la dificultad de afrontamiento, búsqueda de apoyo profesional y evitación emocional.

\section{Objetivos}

En el presente estudio se han planteado los siguientes objetivos:

Objetivo general: Comprobar si existe relación entre las estrategias de afrontamiento y la ansiedad.

Objetivos específicos: Analizar si existen diferencias en el uso de estrategias de afrontamiento adaptativas o activas y estrategias de afrontamiento desadaptativas o pasivas, entre la muestra clínica diagnosticada de ansiedad y la muestra no clínica sin diagnóstico. Analizar si hay diferencias en las siguientes estrategias entre ambos grupos: resolución de problemas, reestructuración cognitiva, apoyo social, expresión emocional, evitación de problemas, pensamiento desiderativo, retirada social y autocrítica. Analizar si en el grupo clínico, existen diferencias en el uso de las estrategias de afrontamiento, en función de si presentan sintomatología ansiosa o además presentan sintomatología depresiva.

\section{Hipótesis}

En función de los objetivos, establecemos las siguientes hipótesis:

Existirán diferencias en el uso de las estrategias de afrontamiento adaptativas o activas y estrategias de afrontamiento desadaptativas o pasivas, entre la muestra clínica diagnosticada de ansiedad y la no clínica sin diagnóstico.

Se observarán diferencias, entre ambos grupos, en el uso de las estrategias de: resolución de problemas, reestructuración cognitiva, apoyo social, expresión emocional, evitación de problemas, pensamiento desiderativo, retirada social y autocrítica. 
Se encontrarán diferencias en el uso de las estrategias de afrontamiento entre el grupo clínico con sintomatología ansiosa y el grupo clínico con sintomatología ansiosa y depresiva.

\section{MÉTODO}

\section{Participantes}

La muestra está compuesta de dos grupos de poblaciones: población clínica (PC) y población no clínica (PNC). La población clínica la conforman pacientes usuarios del servicio de atención psicológica del Centro de Salud Mental de Lorca. La población no clínica está compuesta de personas que no reciben atención psicológica. El muestreo fue incidental.

Los criterios de inclusión para el primer grupo son: ser mayor de edad, presentar uno o más diagnósticos de ansiedad según la CIE-10 (Organización Mundial de la Salud, OMS, 1992), del F.40 al F.48, y ser paciente del Centro de Salud Mental de Lorca.

Los criterios de inclusión para el segundo grupo son: ser mayor de edad, no estar diagnosticado de un trastorno de ansiedad y no estar recibiendo, por ello, atención psiquiátrica ni psicológica en el momento del estudio.

La muestra total es de 73 personas, 35 del grupo PC y 38 del grupo PNC. En la tabla 1 se muestra el perfil sociodemográfico de cada uno de los grupos.

\begin{tabular}{|c|c|c|c|c|}
\hline \multirow[t]{2}{*}{ Variable } & \multicolumn{2}{|c|}{ Frecuencia } & \multicolumn{2}{|c|}{ Porcentaje } \\
\hline & $\mathrm{PC}$ & PNC & $\mathrm{PC}$ & PNC \\
\hline \multicolumn{5}{|l|}{ Sexo } \\
\hline Masculino & 12 & 16 & 34.3 & 42.1 \\
\hline Femenino & 23 & 22 & 65.7 & 57.9 \\
\hline \multicolumn{5}{|r|}{ Edad } \\
\hline $18-34$ & 10 & 19 & 28.6 & 50.0 \\
\hline $35-50$ & 18 & 11 & 51.4 & 28.9 \\
\hline 51 o más & 7 & 8 & 20.0 & 21.1 \\
\hline \multicolumn{5}{|l|}{ Estado civil } \\
\hline Soltero & 13 & 20 & 37.1 & 52.6 \\
\hline Casado & 15 & 14 & 42.9 & 36.8 \\
\hline Divorciado & 3 & 2 & 8.6 & 5.3 \\
\hline Viudo & 1 & 0 & 2.9 & 0 \\
\hline Unión libre & 3 & 2 & 8.6 & 5.3 \\
\hline \multicolumn{5}{|l|}{ Nivel de estudios } \\
\hline Sin estudios & 0 & 1 & 0 & 2.6 \\
\hline Primarios & 13 & 8 & 37.1 & 21.1 \\
\hline Secundarios & 7 & 2 & 20.0 & 5.3 \\
\hline Bachillerato/FPmedio & 5 & 2 & 14.3 & 5.3 \\
\hline Superiores & 10 & 25 & 28.6 & 65.8 \\
\hline
\end{tabular}

En la PC los participantes fueron el $34.3 \%$ hombres y el $65.7 \%$ mujeres, siendo en la PNC los porcentajes de $42.1 \%$ para hombres y $57.9 \%$ para mujeres. En relación a la edad, para el rango de entre 18 y 34 años, la PC ofrece un $28.6 \%$ y la PNC un 50\%, entre 
35 y 50 años encontramos en la PC un $51.4 \%$ y en la PNC un $28.9 \%$ y con 51 años o más, la PC cuenta con un $20 \%$, mientras que la PNC muestra un $21.1 \%$.

Con respecto al estado civil, la categoría de soltero la presentan en la PC un $37.1 \%$ de personas y en la PNC un $52.6 \%$, un $42.9 \%$ de la PC y un $36.8 \%$ de la PNC están casados, un $8.6 \%$ de la PC y un $5.3 \%$ de la PNC están divorciados, un $2.9 \%$ viudo en PC y ninguno en PNC. Y, por último, de la PC un $8.6 \%$ están en unión libre, siendo el porcentaje en PNC de un 5.3\%.

En el grupo de PC, los pacientes presentan diagnósticos según la CIE-10 (OMS, 1992) de ansiedad, agrupados en el apartado de Trastornos neuróticos que oscilan entre el F.41 y el F.45. Los diagnósticos de los pacientes se pueden agrupar en las siguientes clasificaciones: 18 en F.41, 2 en F.42, 14 en F.43, y 5 en F.45. Además, existen cuatro casos en los que aparece comorbilidad de dos trastornos de ansiedad. Y en otros cuatro casos existe una comorbilidad con otro trastorno que no es de ansiedad.

\section{Instrumentos}

Se administraron los siguientes instrumentos:

Un cuestionario sociodemográfico, en el que se recogía información sobre las variables sexo, edad, estado civil y nivel de estudios.

Para valorar los niveles de ansiedad y depresión, se empleó la Escala de Ansiedad y Depresión de Goldberg (EADG; Goldberg, Bridges, Duncan-Jones, y Grayson, 1988), en su versión adaptada al español por Montón, Echevarría, y Campos (1993). Esta escala se utiliza para detectar signos y síntomas de ansiedad y de depresión, en contextos asistenciales de Atención Primaria y epidemiológicos. Se trata de una herramienta de screening que detecta "casos probables", es heteroadministrada y está dividida en dos subescalas, una de ansiedad y otra de depresión, de 9 ítems cada una. La respuesta es dicotómica, contestando si ha estado presente o no cada uno de los síntomas de cada ítem durante las últimas dos semanas. Los puntos de corte se sitúan en 4 o más para la ansiedad y 2 o más para la depresión, a partir de donde se considera que es probable que exista un trastorno, y a mayor puntuación de mayor gravedad. La sensibilidad del instrumento es del 83\% y la especificidad del 82\% (Montón et al., 1993). Arrieta, Díaz, y González (2014) obtuvieron una consistencia interna del instrumento con un alfa de Cronbach de 0.81 para la subescala de ansiedad y de 0.77 para la escala de depresión. Por otro lado, en el estudio llevado a cabo por Espinosa, Orozco, e Ybarra (2015), se halló un alfa de Cronbach de 0.85 para la subescala de ansiedad y de 0.83 para la subescala de depresión. Todas las puntuaciones se interpretan como buenos niveles de fiabilidad.

Por último, con el objetivo de evaluar las estrategias de afrontamiento, se administró el Inventario de Estrategias de Afrontamiento, creado originalmente por Tobin et al. (1989) y adaptado al español por Cano et al. (2007). Presenta una estructura jerárquica con ocho escalas primarias, cuatro secundarias y dos terciarias. Primeramente, 
la persona describe una situación estresante y después contesta cada uno de los 40 ítems de los que está compuesta la prueba, con una escala tipo Likert de intensidad, que va desde 0 "En absoluto", pasando por 1 "Un poco", 2 "Bastante", 3 "Mucho", hasta 4 "Totalmente". Al final aparece otro ítem que evalúa la autoeficacia percibida del afrontamiento. Las ocho escalas primarias son las siguientes (Cano et al., 2007):

Resolución de problemas: estrategias de tipo cognitivo y conductual que modifican la situación generadora de estrés para reducir el mismo.

Reestructuración cognitiva: estrategias de tipo cognitivo que cambian el significado dado a la situación estresante.

Apoyo social: estrategias relacionadas con la búsqueda de apoyo emocional a través de otras personas.

Expresión emocional: estrategias relacionadas con la liberación de emociones que surgen durante la situación estresante.

Evitación de problemas: estrategias relacionadas con la negación y la evitación, tanto de actos como de pensamientos, de la situación estresante.

Pensamiento desiderativo: estrategias de tipo cognitivo que expresan el deseo de que la realidad no fuera estresante.

Retirada social: estrategias relacionadas con el aislamiento de amigos, familiares y compañeros que se asocian a la reacción emocional de la situación estresante.

Autocrítica: estrategia relacionada con el proceso de culpabilizarse y autocriticarse porque ocurriera la situación estresante o por no manejarla adecuadamente.

Las cuatro escalas secundarias son una agrupación empírica de las primarias: manejo adecuado centrado en el problema (resolución de problemas y reestructuración cognitiva), manejo adecuado centrado en la emoción (apoyo social y expresión emocional), manejo inadecuado centrado en el problema (evitación de problemas y pensamiento desiderativo) y manejo inadecuado centrado en la emoción (retirada social y autocrítica). Y las dos escalas terciarias resultan de la agrupación de las segundas: manejo adecuado (resolución de problemas, reestructuración cognitiva, apoyo social y expresión emocional) el cual indica un afrontamiento de tipo activo y adaptativo y manejo inadecuado (evitación de problemas, pensamiento desiderativo, retirada social y autocrítica), indicando éstas un afrontamiento de tipo pasivo y desadaptativo (Cano et al., 2007). En la investigación de Cano et al. (2007), en la que adaptan el inventario al español, se obtuvieron unos coeficientes de consistencia interna entre 0.63 y 0.89 en alfa de Cronbach.

\section{Procedimiento}

Una vez obtenida la aprobación de la investigación por el Comité de Investigación del Área III del Servicio Murciano de Salud (Hospital General Universitario 
Rafael Méndez de Lorca) se procedió a administrar las pruebas a los pacientes. Simultáneamente también se administró a la población no clínica.

Junto con los instrumentos de evaluación, se incluía un consentimiento informado y una hoja informativa donde se explicaba la investigación, el objetivo de la misma, en qué consistía la participación de la persona y la confidencialidad. Cada uno de los participantes firmó un consentimiento informado por escrito como acuerdo de su participación en el estudio.

Tras la obtención de la información necesaria de los participantes del estudio, se llevaron a cabo los análisis de datos.

Se trata de un diseño ex post facto prospectivo simple, descriptivo-comparativo.

Se ha utilizado el programa estadístico IBM SPSS Statistics 24 (IBM Corp., 2016) para analizar los datos. Mediante el análisis de frecuencias, se calcularon las frecuencias y porcentajes de las variables sociodemográficas de cada muestra. Seguidamente, con el análisis descriptivo, se averiguaron las medias y desviaciones típicas de las variables medidas, para cada muestra, por la Escala de Ansiedad y Depresión de Goldberg (Montón et al., 1993) y el Inventario de Estrategias de Afrontamiento (Cano et al., 2007). Por otro lado, en cuanto al análisis inferencial, se llevó a cabo una comparación de medias de muestras independientes con las puntuaciones del Inventario de Estrategias de Afrontamiento, para averiguar si existían diferencias significativas entre ambas muestras, a través de la prueba “ $t$ " de Student. Además, se utilizó la prueba U de MannWhitney para muestras independientes.

\section{RESULTADOS}

Las puntuaciones medias obtenidas por el grupo de PC en ansiedad y depresión fueron de 6.09 y 3.40 , respectivamente. Por su parte, la PNC obtuvo unas medias de 3.11 para ansiedad y 1.21 para depresión.

En cuanto al afrontamiento, como se observa en la tabla 2, el conjunto de estrategias adaptativas o activas, que agrupa: resolución de problemas, reestructuración cognitiva, apoyo social y expresión emocional, fue empleado menos en la PC que en la PNC (PC: $M=42.46, D T=14.975$; PNC: $M=49.53, D T=14.243$ ).

$\mathrm{Y}$ el conjunto de estrategias pasivas, que agrupa: evitación de problemas, pensamiento desiderativo, retirada social y autocrítica, fue utilizado más en la PC que en la PNC (PC: $M=33.63, D T=12.233$; PNC: $M=32.45, D T=10.575$ ).

Analizando las puntuaciones medias de cada estrategia, la PC empleó menos que la PNC las estrategias de: resolución de problemas (PC: $M=12.94, D T=6.092$; PNC: $M=14.26, D T=4.808$ ), reestructuración cognitiva (PC: $M=8.57, D T=5.147$; PNC: $M=12.08, D T=4.148$ ), apoyo social (PC: $M=11.03, D T=5.437$; PNC: $M=13.00$, 
$D T=5.225$ ), expresión emocional (PC: $M=9.91, D T=5.607$; PNC: $M=10.18, D T=5.680$ ) y autocrítica (PC: $5.00, D T=5.891$; PNC: $M=6.37, D T=5.664$ ).

Por otro lado, la PC puntuó más alto que la PNC en las estrategias de: evitación de problemas (PC: $M=6.66, D T=5.423$; PNC: $M=6.24, D T=4.110$ ), pensamiento desiderativo (PC: $M=15.06, D T=5.407$; PNC: $M=14.32, D T=4.479$ ) y retirada social (PC: $M=6.91, D T=5.243$; PNC: $M=6.05, D T=3.869$ ).

En relación al análisis inferencial, al comparar las medias de las estrategias de afrontamiento en ambos grupos, se encontraron diferencias significativas en las medias de: reestructuración cognitiva $(p=.002)$ y estrategias activas $(p=.042)$. En el resto de estrategias, así como en el conjunto de estrategias pasivas, se encontraron diferencias en las medias, pero éstas no llegaron a ser significativas.

Tabla 2. Estadísticos descriptivos y comparación de medias de ambas poblaciones

\begin{tabular}{|c|c|c|c|c|c|}
\hline Estrategia de afrontamiento & Población & Media & Desviación típica & Prueba $t$ & Sig. Prueba $t$ \\
\hline \multirow{2}{*}{ Resolución de problemas } & Clínica & 12.94 & 6.092 & \multirow{2}{*}{-1.032} & \multirow{2}{*}{.306} \\
\hline & No clínica & 14.26 & 4.808 & & \\
\hline \multirow{2}{*}{ Reestructuración cognitiva } & Clínica & 8.57 & 5.147 & \multirow{2}{*}{-3.217} & \multirow{2}{*}{$.002 *$} \\
\hline & No clínica & 12.08 & 4.148 & & \\
\hline \multirow{2}{*}{ Apoyo social } & Clínica & 11.03 & 5.437 & \multirow{2}{*}{-1.580} & \multirow{2}{*}{.119} \\
\hline & No clínica & 13.00 & 5.225 & & \\
\hline \multirow{2}{*}{ Expresión emocional } & Clínica & 9.91 & 5.607 & \multirow{2}{*}{-.204} & \multirow{2}{*}{.839} \\
\hline & No clínica & 10.18 & 5.680 & & \\
\hline \multirow{2}{*}{ Evitación de problemas } & Clínica & 6.66 & 5.423 & \multirow{2}{*}{.371} & \multirow{2}{*}{.712} \\
\hline & No clínica & 6.24 & 4.110 & & \\
\hline \multirow{2}{*}{ Pensamiento desiderativo } & Clínica & 15.06 & 5.407 & \multirow{2}{*}{.640} & \multirow{2}{*}{.524} \\
\hline & No clínica & 14.32 & 4.479 & & \\
\hline \multirow{2}{*}{ Retirada social } & Clínica & 6.91 & 5.243 & \multirow{2}{*}{.793} & \multirow{2}{*}{.431} \\
\hline & No clínica & 6.05 & 3.869 & & \\
\hline \multirow{2}{*}{ Autocrítica } & Clínica & 5.00 & 5.891 & \multirow{2}{*}{-1.012} & \multirow{2}{*}{.315} \\
\hline & No clínica & 6.37 & 5.664 & & \\
\hline \multirow{2}{*}{ Estrategias activas } & Clínica & 42.46 & 14.975 & \multirow{2}{*}{-2.067} & \multirow{2}{*}{$.042^{*}$} \\
\hline & No clínica & 49.53 & 14.243 & & \\
\hline \multirow{2}{*}{ Estrategias pasivas } & Clínica & 33.63 & 12.233 & \multirow{2}{*}{.442} & \multirow{2}{*}{.660} \\
\hline & No clínica & 32.45 & 10.575 & & \\
\hline
\end{tabular}

Además, se realizó una comparación de resultados con la PC. Ésta se dividió en dos grupos, un grupo estaba formado por los pacientes que según la subescala de depresión de la Escala de Ansiedad y Depresión de Goldberg no presentaban sintomatología depresiva acorde con un probable trastorno depresivo, y el otro grupo estaba formado por pacientes que obtuvieron puntuaciones en depresión acordes a un probable trastorno depresivo. El criterio se definió de la siguiente forma: en el primer grupo, la depresión puntuaba con 0 o 1 punto, y en el segundo grupo, la depresión puntuaba con 2 o más puntos.

Los resultados mostraron que no se encontraron diferencias significativas en el uso de cada una de las estrategias de afrontamiento en particular, ni en el conjunto que 
agrupa a las estrategias activas. Sí que se encontraron diferencias significativas en el conjunto que agrupa las estrategias de afrontamiento pasivo, empleando significativamente más la PC con sintomatología depresiva estas estrategias que la PC sin sintomatología depresiva (U de Mann-Whitney: 105.500; Sig: .049).

\section{DISCUSIÓN Y CONCLUSIONES}

En primer lugar, atendiendo a las puntuaciones medias obtenidas por los participantes en la Escala de Ansiedad y Depresión de Goldberg, destacamos la puntuación en depresión por parte de la PC, superando el punto de corte, y dando positivo en "probable caso" de depresión. Estos resultados se relacionan con la comorbilidad que existe entre los trastornos de ansiedad y de depresión, para lo que Brown y Barlow (2009) ofrecen el dato de que el $55 \%$ de los pacientes con ansiedad o depresión, presentan simultáneamente otro trastorno de ansiedad o depresión.

Aunque los pacientes de esta investigación no están diagnosticados de un trastorno depresivo, algunos de ellos presentan sintomatología depresiva. Resulta relevante conocer este dato, ya que la depresión comórbida a un trastorno de ansiedad supone que éste persista más en el tiempo, además de que aumenta la demanda de servicios sanitarios, a diferencia de la presencia única de un trastorno de ansiedad (Hunt, Slade, y Andrews, y Tylee, citados en Clark y Beck, 2012).

En cuanto a la primera hipótesis, es destacable la diferencia significativa hallada en la comparación de las estrategias activas o adaptativas. Estas estrategias se relacionan con acciones encaminadas a solventar la situación de estrés y se pueden denominar también de manejo adecuado (Tobin et al., 1989), por lo que señalamos que los pacientes que tienen un trastorno de ansiedad emplean, menos que la población no clínica, las estrategias que se orientan a enfrentar el problema, buscar apoyo social, expresar las emociones y reevaluar el evento estresante.

Tal y como indica Lazarus (2000), el estrés aumenta si el afrontamiento es inefectivo, en este caso, el bajo empleo de las estrategias adaptativas de una población respecto a otra, podría explicar los niveles más altos de estrés de una población respecto a otra. Este dato coincide con el de Jauregui et al. (2016) que encontraron relación con un nivel bajo de ansiedad y un mayor uso de estrategias adaptativas.

Estos autores, citan a González-Barrón, Montoya-Castilla, Casullo, y Bernabéu-Verdú, para señalar que precisamente el uso de este tipo de estrategias adaptativas puede influir sobre el bienestar psicológico.

Además, esto concuerda con los resultados de Gantiva et al. (2010) y Sandín y Chorot (2003), que encontraron que el grupo de personas con ansiedad, a diferencia del grupo sin ansiedad, empleaba menos estrategias centradas en la solución del problema y en la reevaluación, siendo éstas estrategias adaptativas. 
Atendiendo a la segunda hipótesis, encontramos diferencias significativas en el uso de la estrategia de reestructuración cognitiva. Esta estrategia se evaluaba con ítems del tipo: "Repasé el problema una y otra vez en mi mente y al final vi las cosas de una forma diferente" o "Cambié la forma en que veía la situación para que las cosas no parecieran tan malas" (Cano et al., 2007).

El uso de esta estrategia supone una modificación del significado de la situación estresante, es decir, una reevaluación. Cuando reevaluamos o revalorizamos una amenaza, la emoción que nos producía inicialmente la situación estresante cambia, y la ansiedad inicial disminuye (Clark y Beck, 2012). La persona con ansiedad realiza una valoración primaria de la situación estresante, como una amenaza y una evaluación secundaria de su capacidad para afrontarla, negativa (Lazarus, 2000), lo cual estaría estrechamente relacionado con el uso escaso de la reestructuración cognitiva.

La población clínica emplea significativamente menos esta estrategia, lo que se asociaría con su elevado nivel de ansiedad. Coincidimos con Gantiva et al. (2010), que encontraron que las personas no diagnosticadas de ansiedad empleaban significativamente más la estrategia de reevaluación positiva. Por otro lado, Casari et al. (2014), hallaron relaciones significativas entre una baja ansiedad y el uso de la estrategia de evaluación positiva, estrategia relacionada con la reestructuración cognitiva.

En cuanto a la tercera hipótesis, la población clínica que no presenta sintomatología depresiva, muestra diferencias en el uso del afrontamiento, respecto de la población clínica que sí que presenta sintomatología depresiva. En concreto, la diferencia significativa se ha encontrado en el conjunto de estrategias pasivas, aquellas que se caracterizan por la evitación del problema, tanto cognitiva como conductual, y el retraimiento. La sintomatología depresiva, junto a la ansiosa, se relacionaría entonces con el uso de las estrategias pasivas o desadaptativas.

Para explicar esto, podemos referirnos a los síntomas que conforman los estados depresivos (APA, 2013): estado de ánimo deprimido, disminución del interés por las actividades, pérdida de energía y de concentración, entre otras. Esto se encontraría asociado con las estrategias de aislamiento social y autocrítica, dos de las estrategias que conforman el grupo de desadaptativas. Jauregui et al. (2016) hallaron relaciones significativas entre la sintomatología depresiva y el uso de las estrategias de manejo inadecuado, desadaptativo o pasivo.

Atendiendo a las medias de uso de las estrategias de cada grupo, aunque no son diferencias significativas, el grupo clínico empleó menos las estrategias adaptativas (resolución de problemas, apoyo social, reestructuración cognitiva y expresión emocional) y más las estrategias desadaptativas (evitación del problema, retirada social y pensamiento desiderativo); con la excepción de que el grupo no clínico empleó más una estrategia desadaptativa, que es la autocrítica. 
Las cuatro estrategias más utilizadas por cada uno, encontramos que son las mismas: pensamiento desiderativo, resolución de problemas, apoyo social y reestructuración cognitiva. Esto indica formas de afrontamiento en común entre ambas poblaciones, estableciéndose la diferencia en la intensidad con la que emplean cada estrategia.

Algo parecido refieren Gantiva et al. (2010), encontrando elementos comunes en el afrontamiento de personas con y sin ansiedad, y situando la diferencia en la frecuencia de uso de cada estrategia. Es destacable el elevado uso de la estrategia de pensamiento desiderativo por ambas poblaciones, por lo que hemos considerado que ésta no es una estrategia discriminante del afrontamiento para comparar los grupos.

Podemos concluir que las estrategias de las personas con ansiedad no se caracterizan por ser adaptativas. Especialmente, estas personas emplean mucho menos que las personas sin trastorno de ansiedad la estrategia de reestructuración cognitiva, estrategia adaptativa que se considera clave a la hora de reevaluar la situación estresante. Los resultados del estudio relacionados con esta estrategia sugieren una especial atención a la reestructuración cognitiva como técnica terapéutica.

Dicha técnica se basa en modificar las creencias y evaluaciones que la persona realiza sobre la amenaza (Clark y Beck, 2012). De esta forma, se confirma la importancia de este tipo de intervención clave para trastornos de ansiedad, de intervenir sobre la evaluación primaria relacionada con la gravedad y probabilidad de la amenaza, y la evaluación secundaria, relacionada con la confianza en la propia capacidad de afrontamiento.

Gantiva et al. (2010) también proponen, en base a sus resultados, la relevancia del empleo de dicha técnica cognitiva. Paralelamente al empleo de la reestructuración cognitiva en la intervención terapéutica, se destaca la relevancia de promover el empleo de otras estrategias adaptativas que permitan reducir los niveles de ansiedad, como son el apoyo social, la expresión emocional y la solución de problemas.

Conociendo estos resultados, resaltamos la necesidad de seguir investigando sobre el funcionamiento del afrontamiento en la ansiedad, con el objetivo de establecer unos criterios que enriquezcan la elaboración de la prevención e intervención terapéutica. Una de las razones del interés por esta línea es la elevada prevalencia de este tipo de trastornos, de los pacientes de un centro de salud mental, el 40,9\% presentaban algún diagnóstico de ansiedad (Ponce et al., 2018).

Por otro lado, nos referimos también a la sobrecarga sanitaria que produce este tipo de patología, tal y como indican Clark y Beck (2012), del total de visitas al médico familiar en un centro sanitario universitario, en el $30 \%$ era mencionada la ansiedad. Y además del coste económico directo por la atención sanitaria, cabe destacar el indirecto, derivado de la pérdida de productividad laboral. 
Estos autores señalan las consecuencias perjudiciales de las estrategias de afrontamiento relacionadas con la preocupación, la búsqueda de seguridad, la evitación del pensamiento y la evitación experiencial; y los efectos de las mismas sobre los estados de salud, destacando la necesidad de incluir herramientas terapéuticas que modifiquen estas estrategias en el tratamiento de la ansiedad clínica.

En lo referente a las limitaciones de este estudio, en primer lugar, el tamaño muestral. Es posible que muchas de las diferencias de medias que no llegan a ser significativas, con una muestra más amplia, ofrezcan dichos resultados. Por otro lado, el uso de un muestreo incidental no garantiza la representatividad, lo que un muestreo aleatorio sí conseguiría. Además, un aspecto que enriquecería el contenido de los resultados podría ser establecer diferencias en el uso de las estrategias de afrontamiento en función del tipo de trastorno de ansiedad, lo que aquí ha sido tenido en cuenta de modo global.

\section{REFERENCIAS}

APA (2013). Manual Diagnóstico y Estadístico de los Trastornos Mentales, $5^{a}$ edición. Washington, DC: APA.

Arrieta, M., Díaz, S., y González, F. (2014). Síntomas de depresión y ansiedad en jóvenes universitarios: prevalencia y factores relacionados. Revista Clínica de Medicina de Familia, 7(1), 14-22.

Brown, T.A., y Barlow, D.H. (2009). A proposal for a dimensional classification system based on the shared features of the DSM-IV anxiety and mood disorders: Implications for assessment and treatment. Psychological Assessment, 21(3), 256. doi: 10.1037/a0016608

Cano, F.J., Rodríguez, L., y García, J. (2007). Adaptación española del Inventario de Estrategias de Afrontamiento. Actas Españolas de Psiquiatría, 35(1), 29-39.

Casari, L.M., Anglada, J., y Daher, C. (2014). Estrategias de afrontamiento y ansiedad ante exámenes en estudiantes universitarios. Revista de Psicología (PUCP), 32(2), 243-269.

Clark, D.A. y Beck, A.T. (2012). Terapia cognitiva para trastornos de ansiedad. New York, USA: Descleé de Brouwer.

Espinosa, M.C., Orozco, L.A., y Ybarra, J.L. (2015). Síntomas de ansiedad, depresión y factores psicosociales en hombres que solicitan atención de salud en el primer nivel. Salud Mental, 38(3), 201-208.

Gantiva, C., Luna, A., Dávila, A., y Salgado, M. (2010). Estrategias de afrontamiento en personas con ansiedad. Psychologia, 4(1), 63-70.

Goldberg, D., Bridges, K., Duncan-Jones, P., y Grayson, D. (1988). Detecting anxiety and depression in general medical settings. British Medical Journal, 297(6653), 890-897.

Herrero, M.F. (2016). Programa de la psicoeducación de la ansiedad y entrenamiento en técnicas de relajación: Manual del Terapeuta. España: Bubok.

IBM Corp. (2016). Guía del usuario de IBM SPSS Statistics 24 Core System. New York, USA: IBM.

Jauregui, P., Herrero-Fernández, D., y Estévez, A. (2016). Estructura factorial del "Inventario de estrategias de afrontamiento" y su relación con la regulación emocional, ansiedad y depresión. Behavioral Psychology, 24(2), 319-340.

Lazarus, R. (2000). Estrés y Emoción. Manejo e implicaciones en nuestra salud. Bilbao, España: Descleé de Brouwer. 
Lazarus, R.S., y Folkman, S. (1984). Stress, appraisal, and coping. New York, USA: Springer.

Montón, C., Pérez-Echevarría, M.J., y Campos, R. (1993). Escalas de ansiedad y depresión de Goldberg: una guía de entrevista eficaz para la detección del malestar psíquico. Atención Primaria, 12(6), 345-349.

OMS (1992). CIE-10: Trastornos Mentales y del Comportamiento: Descripciones clínicas y pautas para el diagnóstico. Madrid, España: Meditor.

Ponce, S., Hilario, P.S., y Orteso, F.J. (2018). Prevalencia de los trastornos de ansiedad y del estado de ánimo en el centro de salud mental de Lorca. Trabajo presentado en el V Congreso Internacional en Contextos Psicológicos, Educativos y de la Salud, Madrid, España.

Pozzi, G., Frustaci, A., Tedeschi, D., Solaroli, S., Grandinetti, P., Di Nicola, M., y Janiri, L. (2015). Coping strategies in a sample of anxiety patients: factorial analysis and associations with psychopathology. Brain and Behavior, 5(8).

Sandín, B., y Chorot, P. (2003). Cuestionario de afrontamiento del estrés (CAE): desarrollo y validación preliminar. Revista de Psicopatología y Psicología Clínica, 8(1), 39-53.

Shrestha, R. (2016). Stressful life events and coping strategies among patients with generalized anxiety disorders. Journal of Institute of Medicine, 39(1), 112-118.

Sierra, J.C., Ortega, V., y Zubeidat, I. (2003). Ansiedad, angustia y estrés: tres conceptos. Revista Subjetividades, 3(1), 10-59.

Tobin, D.L., Holroyd, K.A., Reynolds, R.V., y Wigal, J.K. (1989). The hierarchical factor structure of the Coping Strategies Inventory. Cognitive Therapy and Research, 13(4), 343-361.

Recibido: 04 de octubre de 2019

Recepción Modificaciones: 14 de noviembre de 2019

Aceptado: 17 de noviembre de 2019 